\title{
Evidence for strong evolution of the cosmic star formation density at high redshifts ${ }^{\star}$
}

\author{
F. Mannucci ${ }^{1}$, H. Buttery ${ }^{2}$, R. Maiolino ${ }^{3}$, A. Marconi ${ }^{2}$, and L. Pozzetti ${ }^{4}$ \\ 1 INAF - Istituto di Radioastronomia, Largo E. Fermi 5, 50125 Firenze, Italy \\ e-mail: filippo@arcetri.astro.it \\ 2 INAF - Osservatorio Astrofisico di Arcetri, Largo E. Fermi 5, 50125 Firenze, Italy \\ 3 INAF - Osservatorio Astronomico di Roma, via di Frascati 33, 00040 Monte Porzio Catone, Italy \\ 4 INAF - Osservatorio Astronomico di Bologna, via Ranzani 1, 40127 Bologna, Italy
}

Received 7 July 2006 / Accepted 4 September 2006

ABSTRACT

\begin{abstract}
Deep HST/ACS and VLT/ISAAC data of the GOODS-South field were used to look for high-redshift galaxies in the rest-frame UV wavelength range and to study the evolution of the cosmic star-formation density at $z \sim 7$. The GOODS-South area was surveyed down to a limiting magnitude of about $(J+K \mathrm{~s})_{A B}=25.5$, looking for drop-out objects in the $z_{850}^{\prime}$ ACS filter. The large sampled area would allow for the detection of galaxies that are 20 times less numerous and 1-2 mag brighter than similar studies using HST/NICMOS near-IR data. Two objects had initially been selected as promising candidates for galaxies at $z \sim 7$, but were subsequently dismissed and identified as Galactic brown dwarfs through detailed analysis of their morphology and Spitzer colors, as well as through spectroscopic information. As a consequence, we conclude that there are no galaxies at $z \sim 7$ down to our limiting magnitude in the field we investigated. Our non detection of galaxies at $z \sim 7$ provides clear evidence for a strong evolution of the luminosity function between $z=6$ and $z=7$, i.e. over a time interval of only $\sim 170 \mathrm{Myr}$. Our constraints also provide evidence of a significant decline in the total star formation rate at $z=7$, which must be less than $40 \%$ of that at $z=3$ and $40-80 \%$ of that at $z=6$. We also derive an upper limit to the ionizing flux at $z=7$, which is only marginally consistent with what is required to completely ionize the Universe.
\end{abstract}

Key words. Galaxy: formation - Galaxy: evolution - galaxies: high-redshift - galaxies: starburst - cosmology: observations early Universe

\section{Introduction}

The sensitivity of new generation instruments, coupled with 8-10-m class ground-based telescopes and with the HST, allowed impressive progress in observational cosmology during the past few years.

Several surveys aimed at searching for high redshift galaxies have obtained large samples of objects at increasing redshifts: $z \sim 1$ (e.g., Cowie et al. 1996), $z \sim 3$ (Steidel et al. 1996, 2003), $z \sim 4$ (Steidel et al. 1999; Hu et al. 1996), $z \sim 5$ (Hu et al. 1999; Spinrad et al. 1998), $z \sim 6$ (Dawson et al. 2001; Fan et al. 2001; Stanway et al. 2003; Bouwens et al. 2004a, 2006; Dickinson et al. 2004; Mobasher et al. 2005), up to the currently accepted record of $z \sim 6.6$ (Hu et al. 2002; Kodaira et al. 2003; Taniguchi et al. 2005). Observations are approaching the interesting redshift range between $z=6$ and $z=10$ where current cosmological models expect to find the end of the reionization period and the "starting point" of galaxy evolution (e.g., Stiavelli et al. 2004)

The results from all these surveys have had a tremendous impact on our understanding of the cosmic history of star formation and on the reionization epoch (Gunn \& Peterson 1965). As an example, the detection of high-redshift quasars by Fan et al. (2001) allowed the measurement of the fraction of neutral gas at $z \sim 6$ (Becker et al. 2001; Fan et al. 2002; Pentericci et al. 2002).

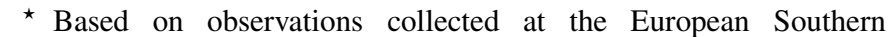
Observatory, Chile, proposals 074.A-0233 and 076.A-0384.
The discovery that this fraction is low but significantly non-zero (nearly the same as or larger than 1\%) puts severe constraints on reionization models. On the contrary, the detection of a high opacity due to ionized hydrogen in the WMAP data (Alvarez et al. 2006) points toward a high reionization redshift $(z \sim 11)$. Thus, detecting objects at even higher redshifts could put strong constraints on the properties of the intergalactic medium at those redshifts (Loeb et al. 2005; Gnedin \& Prada 2004; Ricotti et al. 2004; Cen et al. 2005).

Most high-redshift galaxies from the previous surveys were selected by the dropout technique, i.e., by detecting the spectral break in the UV continuum blueward of the Ly $\alpha$ due to intervening Ly $\alpha$-forest (see, for example, Steidel et al. 2003). This technique is mainly sensitive to high-redshift UV-bright galaxies, commonly named Lyman-Break Galaxies (LBGs). The use of different filters allows the selection of different redshift ranges, from $z \sim 3$ (U-dropout, Steidel et al. 1996, 2003), to $z \sim 6$ (i-dropout, Stanway et al. 2003; Bouwens et al. 2004a, 2006; Shimasaku et al. 2005), $z \sim 7$ ( $z^{\prime}$-dropout, Bouwens et al. 2004b; Bouwens \& Illingworth 2006), and even $z \sim 10$ (J-dropout, Bouwens et al. 2005; Mobasher et al. 2005); see Hopkins (2004) and Hopkins \& Beacom (2006) for two recent reviews.

Up to a redshift of about 6 , galaxies can be detected by using optical data alone, as the Lyman break occurs at $\lambda<0.85 \mu \mathrm{m}$ and the continuum above Ly $\alpha$ can be sampled, at least, by the $z_{850}^{\prime}$ HST/ACS filter. This allowed for the detection of a large number of objects at $z=6$, which provides evidence of strong 
evolution of the Luminosity Function (LF) of the LBGs between $z=6$ and $z=3$. This is described as the brightening, with increasing cosmic age, of the typical luminosity $\left(L^{*}\right)$ of about 0.7 mag (Bouwens et al. 2006) or the increase in the comoving density $\left(\Phi^{*}\right)$ by a factor of 6 (Bunker et al. 2004).

At redshifts higher than 6 the use of near-IR images is mandatory, which makes detection much more difficult. Deep $J$-band images from HST/NICMOS exist, but their field-of-view is limited to a few sq.arcmin. Larger fields can be observed by ground-based telescopes, but at the expense of worse PSFs (about $0.5-1.0^{\prime \prime}$ FWHM instead of $0.4^{\prime \prime}$ for NICMOS/NIC3 and $0.1^{\prime \prime}$ for HST/ACS) and brighter detection limits.

Bouwens at al. (2004b) used one of these deep NICMOS fields in the Hubble UltraDeep Field (HUDF) to detect $z^{\prime}$-dropout objects. They detected 5 objects in a 5.7 sq.arcmin field down to a limiting mag of $H_{A B} \sim 27.5$, showing the possible existence of a reduction in the Star Formation Density (SFD) at $z>6$. Recently, Bouwens \& Illingworth (2006) enlarged this search to $\sim 19$ sq.arcmin using new NICMOS data and improved data reduction. They detected four possible $z \sim 7$ objects, while 17 were expected based on the $z=6 \mathrm{LF}$. Also, they found that at least 2 of the 5 sources in their previous study were spurious. These new results point toward the existence of a strong reduction in the LF with increasing redshift at $z>6$. In contrast, Mobasher et al. (2005), looking for J-dropout galaxies in the HUDF, detected one object that could be a massive post-starburst galaxy at $z \sim 6.5$, formed at high redshift, $z>9$, (but see also Eyles et al. (2006) and Dunlop et al. (2006), who suggest lower redshifts for this object). Deep near-infrared data were also used by Richard et al. (2006) to examine two lensing clusters, and detect eight optical dropouts showing Spectral Energy distributions (SEDs) compatible with high-redshift galaxies. After correcting for lensing amplification, they derived an SFD well in excess of the one at $z=3$, hinting at large amounts of star formation activity during the first Gyr of the universe.

By extrapolating the LF observed at $z \sim 6$ (Bouwens et al. 2006) toward higher redshifts, it is possible to see that those studies based on small-field, deep near-IR data are able to detect only galaxies that are much more numerous, and therefore fainter, than $L^{*}$ galaxies. Their faint magnitudes imply that their redshifts cannot be spectroscopically confirmed with the current generation of telescopes, and it is difficult to distinguish these candidates from the Galactic brown dwarfs with the same colors. As a consequence, these data cannot be used to confidently put strong constraints on the amount of evolution of the LF at $z>6$ : if all the candidates are real star-forming galaxies at the proposed redshift, the SFD at $z=7$ and $z=10$ could be similar to that at $z=6$. If, on the contrary, none of them are real, the upper limits of the SFD would imply a decrease of the SFD toward high redshifts. As a consequence, the evolution of the SFD at $z>6$ is not yet well constrained. The knowledge of this evolution is fundamental for a good understanding of the primeval universe, for example, of the sources of reionization.

Here we present a study aimed at detecting bright $z=7$ objects in a large (unlensed) field, in order to measure the cosmic star-formation density at this redshift. This is important for two main reasons: first, comparing these results for bright objects with those by Bouwens et al. (2004b) and Bouwens \& Illingworth (2006) for fainter $z^{\prime}$-dropouts will allow us to study different part of the LF. This is needed, for example, to distinguish between luminosity and density evolution and to compare it with the dark matter distribution predicted by the cosmological models. Second, the detections of relatively bright objects would allow a more complete study of the properties of these galaxies in terms of morphology, spectroscopy, and SEDs. We will show that the absence of $z \sim 7$ objects with $M(1500)<-21.4$ in the survey field means that the galaxy luminosity function evolved significantly in the short time (about $170 \mathrm{Myr}$ ) between $z=7$ and $z=6$. The epoch at $z=7$, about 750 Myr after the Big Bang, can therefore be considered the beginning of the bulk of star formation.

Throughout this paper we use the concordance cosmology $\left(h_{100}, \Omega_{\mathrm{m}}, \Omega_{\Lambda}\right)=(0.7,0.3,0.7)$ and $\mathrm{AB}$ magnitudes.

\section{Observations and object catalog}

The GOODS-South field, centered on the Chandra Deep Field South (CDFS), is a region of about $10 \times 15$ arcmin that is the subject of deep observations by many telescopes. HST observed it with ACS using the broad-band filters $b_{435}, v_{606}, i_{775}^{\prime}$, and $z_{850}^{\prime}$ (Giavalisco et al. 2004a). Most of the GOODS-South was observed in $J, H$, and $K$ s with the VLT/ISAAC (Labbé et al. 2003; Vandame et al. in preparation; see also Grazian et al. 2006). Many other deep observations are available for the GOODSSouth field. The Spitzer satellite obtained images of this field in all its bands, in particular in the IRAC bands 1 and 2, corresponding to 3.6 and $4.5 \mu \mathrm{m}$.

The main catalog is based on ESO/VLT $J$ and $K$ s data covering $141 \mathrm{sq} \cdot \operatorname{arcmin}^{1}$. The typical exposure time is $3.5 \mathrm{~h}$ in $J$ and $6 \mathrm{~h}$ in $K$, with a seeing of about $0.45^{\prime \prime} F W H M$ (see also Fig. 4). We used the $J+K$ s sum image to build the main object catalog: actively star forming galaxies are expected to have a flat (in $f_{v}$ units) spectrum between $J$ and $K$, and therefore the use of the combined image is expected to improve the object detectability. The program Sextractor (Bertin \& Arnouts 1996) was used to extract the object catalog. We selected objects having flux above 1.2 times the rms of the sky over a $0.22 \mathrm{sq}$.arcsec area (10 contiguous pixels). The part of the images near the edges were excluded as the noise increases because of telescope nodding. The final covered area is 133 sq.arcmin. The average $6 \sigma$ magnitude limit inside a $1^{\prime \prime}$ aperture is 25.5 , estimated from the statistics of the sky noise. This agrees well with the histogram of the detected sources as a function of the $(J+K s)$ magnitude shown in Fig. 1, showing that this magnitude corresponds to $95 \%$ of completeness. The main catalog comprises about 11000 objects.

The colors of the objects were computed from the photometry inside an aperture of $1^{\prime \prime}$ of diameter for the HST/ACS and VLT/ISAAC images.

\section{Candidate selection}

As discussed above, galaxies at $z \sim 7$ can be selected as $z^{\prime}$-dropouts, i.e. objects with very red $z^{\prime}-J$ colors, indicating the presence of a break, and with blue $J-K$ colors, which disentangles high-redshift star-forming galaxies from reddened foreground galaxies. This is illustrated by the $z^{\prime}-J$ versus $J-K$ color diagram in Fig. 2, where we plot the colors expected for different classes of galaxies affected by various amounts of dust reddening (from Bruzual \& Charlot 2003 and Mannucci et al. 2001) along with the data from our sample. The figure shows that objects with both red $z^{\prime}-J$ and red $J-K$ color are likely to be reddened galaxies at intermediate redshift. In Fig. 2 the thick tracks indi-

1 To build the catalog we used the images of the public release version 1.0, while the photometry was computed on the images of the 1.5 version, available from October 2005. 


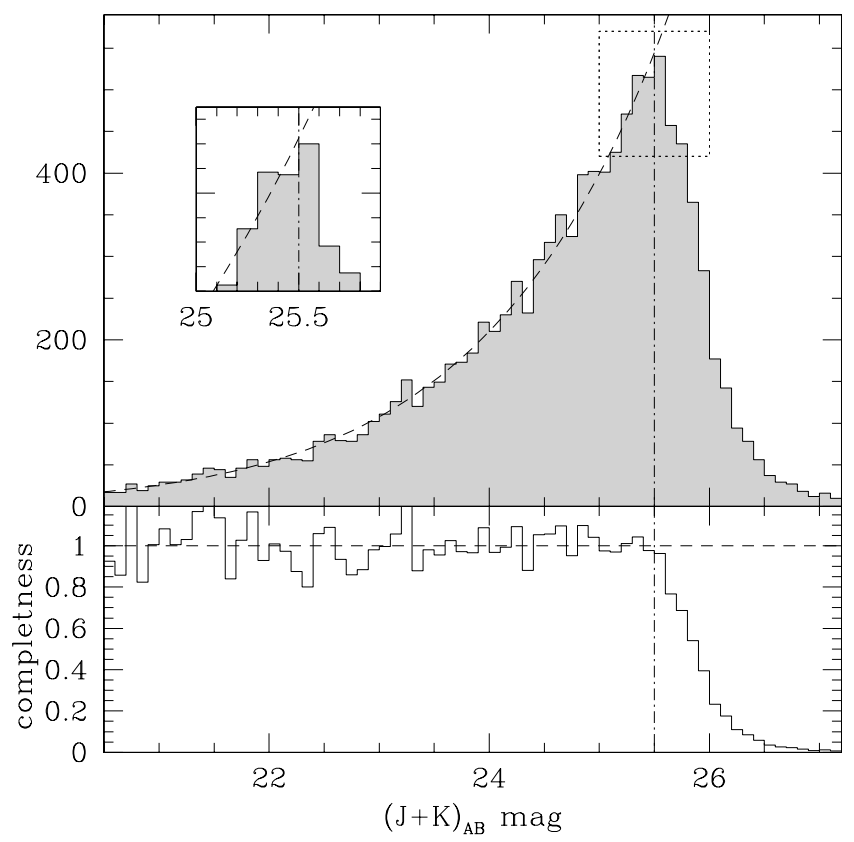

Fig. 1. Upper: histogram of the objects detected in the $(J+K s)$ image. The inserted box shows an enlargement of the dotted square. The dashed line shows a polynomial fit to the histogram. The dot-dashed line shows the used detection limit as derived from the statistics of the sky noise. Lower: completeness of the detections computed as the ratio between the fit and the histogram of the upper panel. The $6 \sigma$ limiting magnitude of $(J+K \mathrm{~s})=25.5$ corresponds to a completeness of about $95 \%$.

cate the colors expected for star forming galaxies at $z>7$, which are indeed separated from reddened galaxies on this diagram.

Another class of possible interlopers are Galactic brown dwarfs. Indeed, these stars can have blue $J-K$ colors and strong methane absorption shortward of $\sim 1 \mu \mathrm{m}$, strongly suppressing the emission in the $z^{\prime}$ band and thus mimicking $z^{\prime}$-dropouts of high redshift galaxies. The coldest brown dwarfs are also undetected in all other optical bands. This is illustrated in Fig. 2, where stars show the colors of brown dwarfs obtained by convolving the spectra in Testi et al. (2001) with the ACS and ISAAC filter transmission curves. These colors extend in the region of the diagram used for selecting of $z^{\prime}$-dropouts. Summarizing, the use of near-IR colors alone is enough to exclude low/intermediate galaxies, but not to distinguish true $z \sim$ 7 star-forming galaxies from high-z QSOs or Galactic brown dwarfs.

We selected objects that are undetected $(<1 \sigma)$ in $i_{775}^{\prime}$ and in all bluer bands and that have $z^{\prime}-J>0.9$ and $J-K \mathrm{~s}<1.2 *$ $\left(z^{\prime}-J\right)-0.28$, as shown in Fig. 2. This region is larger than expected for galaxies at $z>7$, so it is possible that a number of lower-redshift interlopers are present in the catalog. This choice is motivated by not having detected any reliable candidate (see below) and is therefore needed to exclude high-redshift galaxies not being selected because of colors just outside an exceedingly narrow selection area.

Despite the common name of "dropouts", we do not require that our candidates are undetected in $z_{850}^{\prime}$. In fact, $z=7$ sources can be quite luminous in this band for two possible reasons: 1) although the $z_{850}^{\prime}$ cut-on filter convolved with the ACS detector efficiency has a response peaking at $\sim 8800 \AA$, its red wing gathers some flux up to $1.05 \mu \mathrm{m}$, and as a consequence, some flux at or above the Ly $\alpha$ line could be transmitted by the filter (indeed this is predicted by tracks of galaxies at $z>7$ in Fig. 2);

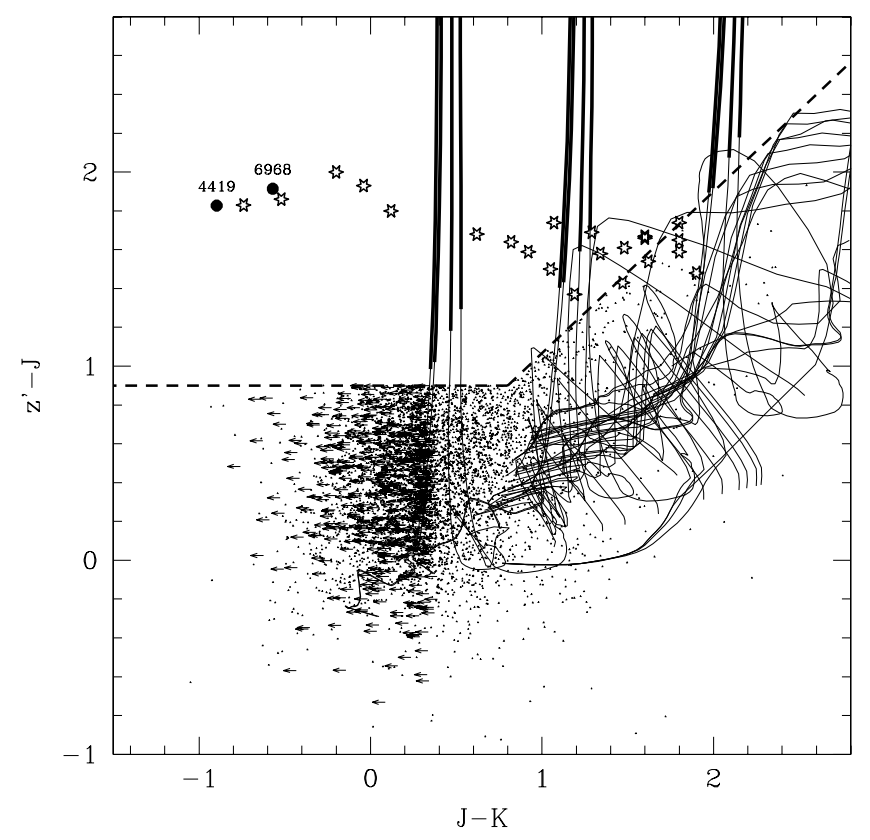

Fig. 2. Color-color selection diagram for $z>7$ galaxies. The solid lines show the variation in the colors with redshift expected for local galaxies (Mannucci et al. 2001) and galaxy models by the Bruzual \& Charlot (2003). Thin and thick lines show the expected colors for galaxies below and above $z=7$, respectively. The three groups of lines correspond to three different amounts of extinctions $(E(B-V)=0.0,0.3$, and 0.6, from left to right), using the Cardelli et al. (1989) extinction law. Stars show the expected positions of Galactic brown dwarfs (ranging from $\mathrm{T} 8$ to L1, from left to right). Small dots show the galaxies detected in the GOODS-South in all three bands, while the arrows show the positions of the objects undetected in $K$. The dashed line shows the color threshold. Above this threshold only objects with no counterparts either in $b_{435}, v_{606}$, and $i_{775}^{\prime}$ or in the sum $b_{435}+v_{606}+i_{775}^{\prime}$ image are shown. Large solid dots show the two objects discussed in Sect. 4. The labels show their entry number in our catalog.

2 ) if the reionization epoch is at $z>7$ (as the recent WMAP results and the spectrum of the galaxy at $z=6.56$ suggest, Bennett et al. 2003; Hu et al. 2002), then the Ly-forest may still have some transmission, similar to what is observed in QSOs at $z \sim 6$ (Pentericci et al. 2002).

An initial catalog was constructed by using the optical photometry from the public GOODS catalog (Giavalisco et al. 2004a) with the aperture photometry computed by Sextractor. This produced the selection of a few tens of $z^{\prime}$-dropouts. These objects were checked by eye to remove spurious detections or objects whose photometry is seriously affected by bright nearby objects. The photometry of the 6 objects that passed this check was measured again by using the IRAF/PHOT program in the same $1^{\prime \prime}$ aperture. Using a local sky computed in an annular region around the object, this program is expected to provide better photometry of faint sources. For 4 of these objects, the new $z^{\prime}-J$ color is much bluer, below the detection threshold. This is an indication that Sextractor overestimated the local sky near these sources.

Two objects remain with colors that are compatible with both star-forming galaxies at $z>7$ and Galactic brown dwarfs. Their images are shown in Fig. 3, while their properties are listed in Table 1 and discussed in the next section. 

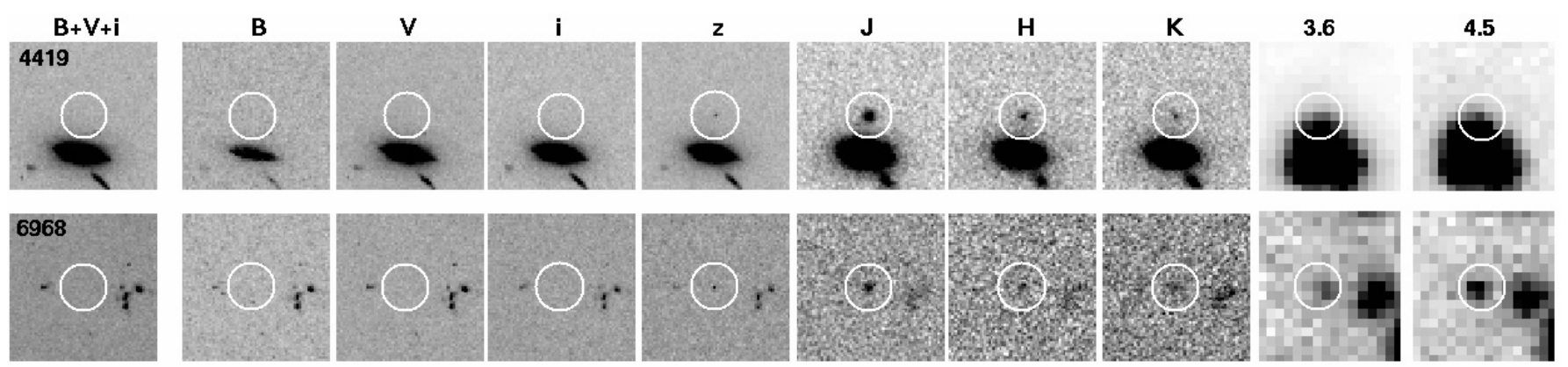

Fig. 3. Images of the two candidates. Each box is 10 arcsec wide. The first panel to the left shows the sum of the optical $b_{435}, v_{606}$ and $i_{775}^{\prime}$ ACS filters. The images in all the filters follow.

Table 1. High-redshift candidates.

\begin{tabular}{cccccc}
\hline \hline ID & \multicolumn{2}{c}{ RA (J2000) Dec } & $z_{850}^{\prime}$ & $J$ & $K \mathrm{~s}$ \\
\hline 4419 & $03: 32: 38.8$ & $-27: 49: 53.7$ & 25.10 & 23.27 & 24.17 \\
6968 & $03: 32: 25.1$ & $-27: 46: 35.7$ & 26.39 & 24.48 & 25.05 \\
\hline
\end{tabular}

\section{The two candidates}

The SED of the two candidates are compatible overall with being high-redshift star-forming galaxies. The 9 available photometric bands, from $b_{435}$ to Spitzer/IRAC $4.5 \mu \mathrm{m}$, can be used to derive photometric redshifts. These two objects are contained in the GOODS-MUSIC catalog (Grazian et al. 2006), object 4409 with ID 7004 and object 6968 with ID 11002 with photometric redshifts of 6.91 and 6.93, respectively. Both objects have already been selected as $i$-dropout objects by other groups. Object 4419 was selected by Stanway et al. (2003) (object SBM03\#5) and further studied by Bunker et al. (2004) (ID number 2140), while object 6968 was selected by Eyles et al. (2006) (ID 33_12465) and by Bouwens \& Illingworth (2006). Both objects were identified as Galactic stars on the basis of the compact ACS morphology and of the $z J K$ colors. In the following we use the morphologies, Spitzer data, and spectra to further investigate the nature of these sources.

Both candidates show very blue $J-K$ colors and have $z^{\prime}-J \sim$ 1.9. This blue $J-K$ color could be due to starburst galaxies or faint AGNs at $z \sim 7$ with a UV spectral slope $\beta$ (with $f_{\lambda} \propto \lambda^{\beta}$ at $\lambda_{\text {rest }}=1500 \AA$ ) that is more negative than -2 . These colors are also typical of Galactic brown dwarf stars of type T6-8, as can be seen in Fig. 2.

In this section we present their properties in terms of the morphology in the optical and near-infrared images (Sect. 4.1), midinfrared colors (Sect. 4.2), and optical spectra (Sect. 4.3).

\subsection{Morphologies}

Both objects are detected in $z_{850}^{\prime}$ and $J$. We studied the morphology of these objects in both bands: the HST/ACS $z_{850}^{\prime}$ images have a much higher resolution (about $0.1^{\prime \prime}$ FWHM), but both objects are much fainter in this band. In contrast, the VLT/ISAAC $J$ band images have a lower resolution (about $0.45^{\prime \prime}$ ), but the higher signal-to-noise ratio per pixel allows a more accurate study. In both cases we compared the object luminosity profile with the PSF derived from a few nearby point sources. As shown in Fig. 4, both objects are consistent with being point sources, as no significant differences are seen with the local PSF.

\subsection{Spitzer colors}

The near- to mid-IR colors can be used to investigate the nature of these objects. Young starburst galaxies at $z \sim 7$ are expected to be intrinsically quite blue and show a flat spectrum above the Lyman limit. We computed the photometry of our candidates in the Spitzer/IRAC $3.6 \mu \mathrm{m}$ and $4.5 \mu \mathrm{m}$ images using $3^{\prime \prime}$ apertures, in order to have aperture corrections similar to the those in the $K$ band, i.e., about 0.42 mag (see, for example, Eyles et al. 2005) and to compare the results with the near-IR data. To compute the photometry of object 4419 , the contribution by the nearby bright galaxy was estimated by convolving the $z_{850}^{\prime}$ band image with the Spitzer PSF. This introduces a large additional uncertainty. Figure 5 shows the $J-K$ vs. $K-4.5 \mu \mathrm{m}$ and 3.6-4.5 $\mu \mathrm{m}$ color-color diagrams, comparing the colors of both candidates with those of the Galactic stars (derived from the brown dwarf models by Allard et al. 2001) and of high-redshift galaxies and type 1 AGNs (Seyfert 1 and QSOs, Francis \& Koratkar 1995). It is evident that these diagrams can be used to distinguish compact galaxies from stars. Even if the Spitzer flux of 4419 is uncertain, because it is affected by the nearby bright galaxy, it appears that both objects show colors compatible with being Galactic stars. The flux in the Spitzer bands of object 6968 was already discussed by Eyles et al. (2006), and they also concluded that they are compatible with being a Galactic brown dwarf.

\subsection{Optical spectra}

The two candidates were observed for $5.0 \mathrm{hr}$ with the FORS2 spectrograph on the ESO VLT in October 2005. The multi-slit facility allowed us to observe the two candidates together with other objects having either very faint counterparts in the $i_{775}^{\prime}$ band or colors above the selection threshold when measured by Sextractor (see Sect. 3). We used 1" wide slits and the G600z grism, providing a dispersion of $1.6 \AA /$ pix and a resolution of about $\lambda / \Delta \lambda=1400$. The covered wavelength range was between 0.75 and $1.05 \mu \mathrm{m}$.

In all cases, no significant emission line and spectral break were detected in this wavelength range. It should be noted that an optical spectrum of object 4419 have already been obtained by Stanway et al. (2004b) with DEIMOS on the Keck-II telescope, but no spectral features were detected.

The emission-line detection limit was computed from the background noise. We obtain a $5 \sigma$ limiting magnitude for unresolved sources of about $3 \times 10^{-18} \mathrm{erg} \mathrm{s}^{-1} \mathrm{~cm}^{-2}$ in the wavelength range $0.900-0.995 \mu \mathrm{m}$, corresponding to a redshift range for the $\operatorname{Ly} \alpha$ line of $6.4<z<7.2$. This limit holds for the part of the wavelength range that is not covered by bright sky lines, which corresponds to about $88 \%$ of the total range. Such a 
4419
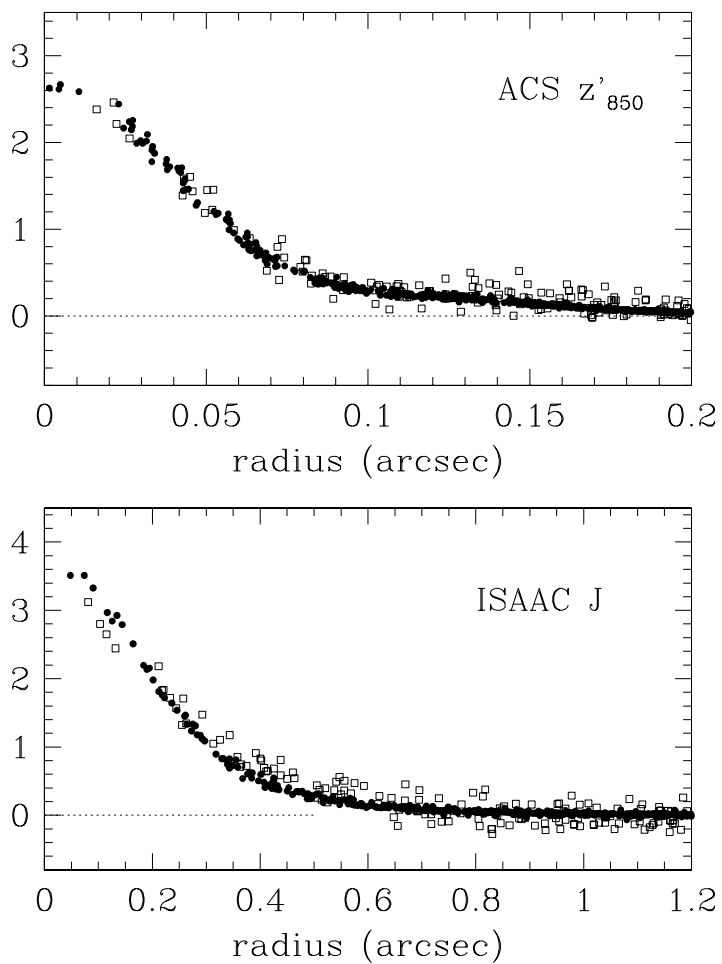

6968
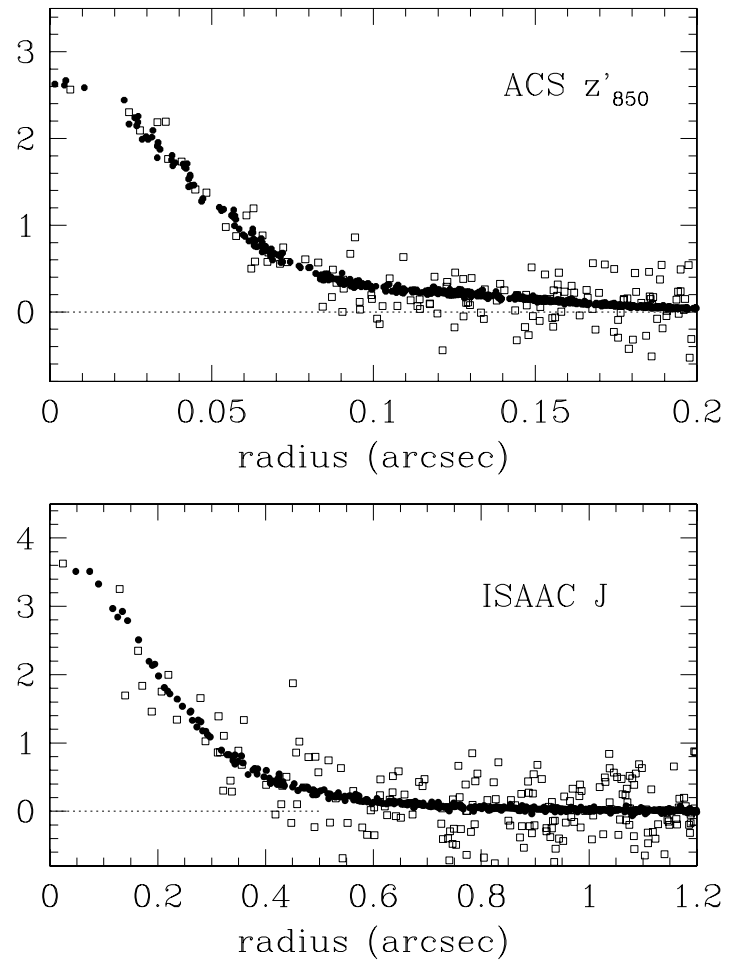

Fig. 4. The observed luminosity profile of candidates 4419 (left) and 6968 (right) are compared with the local PSF in the $z_{850}^{\prime}$ (upper panels) and $J$-band (lower panels) images. Black dots are the PSF derived from 4 nearby stars, the white squares the luminosity profiles of the objects. Both objects are unresolved.

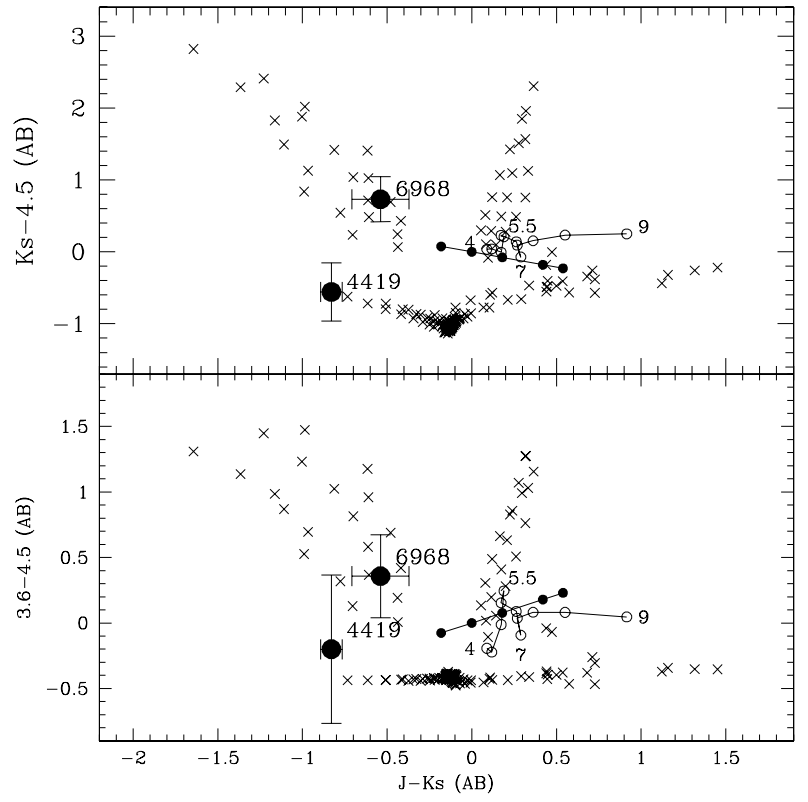

Fig. 5. Near- and mid-infrared Spitzer/IRAC colors of the two candidates (black dots with error bars) compared with the expected colors of Galactic brown dwarfs (crosses, from Allard et al. 2001), starburst galaxies at $z \sim 7$ (connected black dots, representing galaxies with UV spectral slope $\beta=-2.3,-2.0,-1.7,-1.3$, and -1.1 , from left to right) and high redshift Seyfert1/QSOs (connected empty dots, for redshifts between 4 and 9 with steps of 0.5 , see the nearby redshift labels). The two candidates fall in the region occupied by the brown dwarfs.

sensitivity would be more than adequate to detect the $\operatorname{Ly} \alpha$ emission from star forming galaxies at these redshift. This flux can be estimated by assuming that the properties of the $i$-dropout galaxies at $z \sim 6$ also holds at these higher redshifts. The spectroscopic observations of $i$-dropouts (Bunker et al. 2003; Stanway et al. 2004a; Dickinson et al. 2004) have shown that colorselected galaxy have Ly $\alpha$ lines with rest-frame equivalent widths (EWs) of 20-30 $\AA$, corresponding to $2-3 \times 10^{-17} \mathrm{erg} \mathrm{s}^{-1} \mathrm{~cm}^{-2}$ for the faintest of the two candidates, well above the detection limit. It should be noted that line-selected high-redshift galaxies show much larger EWs, on the order of $200 \AA$ (e.g., Malhotra $\&$ Rhoads 2002). Similar values are derived for high redshift AGNs: scaling the Ly $\alpha$ fluxes in $z \sim 6$ quasars observed by Fan et al. (2003) and Maiolino et al. (2004) to the continuum luminosity of the current sample, we would obtain fluxes on the order of $1-4 \times 10^{-17} \mathrm{erg} \mathrm{cm}^{-2} \mathrm{~s}^{-1}$.

As a conclusion, all of the four investigations presented above confirm the identification of both sources as Galactic stars. Also their luminosity is consistent with this identification: if they are brown dwarfs of type from T6 to T8, their absolute mag would be between $M(J)=15.5$ and $M(J)=16.5$ (Leggett et al. 2002), placing them between 200 and 700 pc from us. As a consequence, no $z=7$ object is present in the survey field above our detection limit.

\section{Evolution of the luminosity function and of the cosmic star-formation density}

The detection of no $z>7$ objects in the field can be used to place an upper limit to the LF of these objects. To do this we need to accurately estimate the volume sampled by our survey. Two effects produce the dependence of this volume on the magnitude of the objects. First, fainter objects are detected in the $(J+K \mathrm{~s})$ image only at lower redshifts; second, only lower limits 

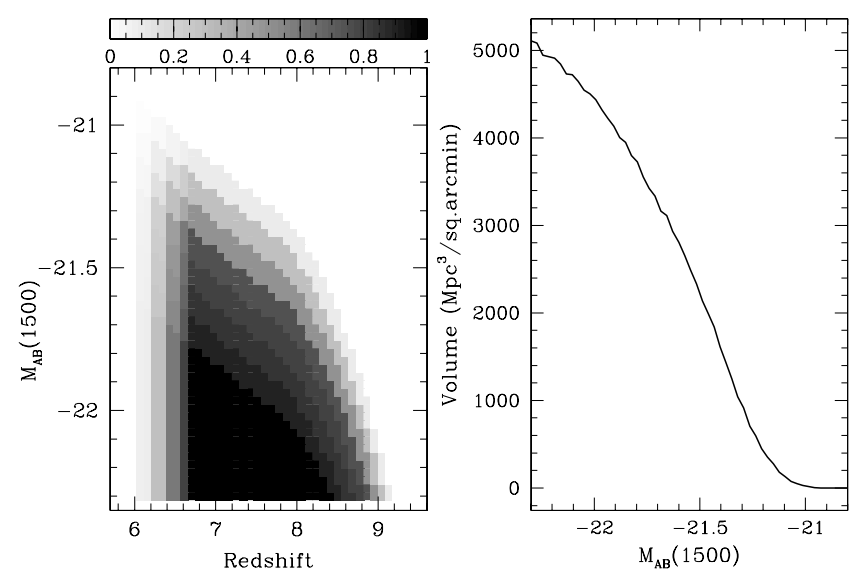

Fig. 6. Left: detection probability for objects of a given absolute magnitude and redshift derived by applying the selection in Sect. 3 and Fig. 2. The color code is described in the bar at the top. Right: the effective comoving volume $V_{\text {eff }}$ sampled at each absolute magnitude by the GOODS observations.

of the $z^{\prime}-J$ color can be measured for objects selected in the $(J+K s)$ image and undetected in $z_{850}^{\prime}$. This lower limit is above the selection threshold only for objects that are bright enough in the $J$ band, while fainter objects can have a color limit below threshold.

The effective sampled volume $V_{\text {eff }}$ can be computed as a function of the absolute magnitude of the objects using

$V_{\text {eff }}(M)=\int p(M, z) \frac{\mathrm{d} V}{\mathrm{~d} z} \mathrm{~d} z$

(Steidel et al. 1999; Stanway et al. 2003), where $p(M, \mathrm{z})$ is the probability of detecting an object of magnitude $M$ at redshift $z$ using the selection in Fig. 2, and $\mathrm{d} V / \mathrm{d} z$ is the comoving volume per unit solid angle at redshift $z$. The results of this computation are shown in Fig. 6.

The detection probability $p(M, z)$ was obtained by computing the expected apparent magnitudes and colors of starburst galaxies (modeled as $f_{\lambda} \propto \lambda^{\beta}$ ) of varying intrinsic luminosity and spectral slope $\beta$, placed at different redshifts. Figure 6 (left panel) shows the results of this computation. The redshift sensitivity of our selection method starts at about $z=6.7$, followed by a peak at $z=7$ and a shallow decrease towards high redshifts. By integrating this function over the redshift, we obtain the results in the right panel of Fig. 6.

The limiting apparent magnitude ( 25.5 , see Sect. 2$)$ corresponds, at $z=7$, to an absolute magnitude $M(1500)=$ -21.4. Using the standard relation between UV luminosity and SFR (Madau et al. 1998), this value corresponds to an SFR of $\sim 20 M_{\odot} / y r$. This value is adequate for sampling the brighter part of the LF of LBG at any redshift. As an example, Stanway et al. (2003) found $9 i$-dropout galaxies at $z \sim 6$ with typical SFRs of 20-30 $M_{\odot} / \mathrm{yr}$, while the SFR of $L^{*}$ galaxies in Bouwens et al. (2006) is $9 M_{\odot} / \mathrm{yr}$. As a reference, the SFR corresponding to an $L^{*}$ galaxy in the $z \sim 3$ sample by Steidel et al. (1996) is about 15-20 $M_{\odot} / \mathrm{yr}$ (Giavalisco 2002; Stanway et al. 2003).

This volume can be used to estimate an upper limit to the density of objects at this redshift range. The limiting density for a given Confidence Level (CL) is given by

$\rho(M, C L)=N(C L) / V_{\mathrm{eff}}(M)$

where $V_{\text {eff }}(M)$ is given by Eq. (1) and $N(C L)$ is the maximum number of objects in the field corresponding to the limiting density at the chosen CL $(2.3$ objects for $C L=90 \%)$. Figure 7 shows

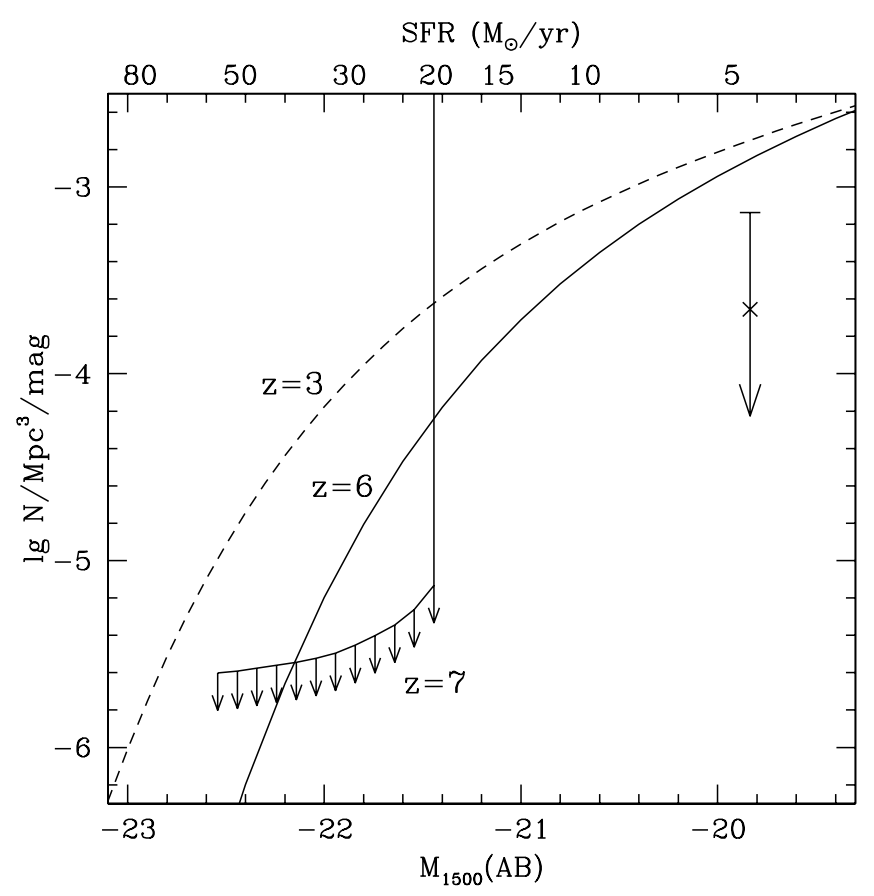

Fig. 7. The limits to the density of $z=7$ galaxies (connected downward arrows) are compared with the LFs of LBGs at $z=3$ (dashed line, Steidel et al. 2003) and $z=6$ (solid line, Bouwens et al. 2006, assuming $M(1350)-M(1500)=0.10$, as for the typical $z=3$ LBGs). The upper limit with a cross corresponds to the object detected by Bouwens et al. (2004b) in the HUDF and considered to be at $z \sim 7$. Data are plotted as a function of the absolute magnitude $M(1500)$ at $1500 \AA$, while the upper axis shows the corresponding value of the SFR.

the $1 \sigma$ upper limits to the density of objects, compared with the LF of the LBGs at $z=6$ from Bouwens et al. (2006) (a Schechter function with parameters $M^{*}(1350)=-20.25, \alpha=-1.73$, and $\left.\Phi^{*}=0.00202 \mathrm{Mpc}^{-3}\right)$ and at $z=3$ obtained by Steidel et al. (2003). The LF at $z=6$ is still subject to large uncertainties because it is based on a compilation of $500 i$-dropouts coming from various fields with different limiting magnitudes. As a consequence, cosmic variance could have different effects in different parts of the LF. The faint-end slope is particularly uncertain, as it is based on faint objects observed at low signal-to-noise. At this signal level, many interlopers at lower redshift could be present in the sample, with the uncertainty on the $i-z$ color causing galaxies to scatter into the selection region, hence overestimating the number of faint sources. It should also be noted that the VVDS collaboration (Le Fevre et al. 2005; Paltani et al. 2006) studied the LF of the high- $z$ galaxy population with $3<$ $z<4$ using a purely magnitude-selected spectroscopic sample, and found an unexpectedly large number of very bright galaxies, pointing toward the possibility that the color selection could be effected by large incompleteness.

Besides these uncertainties, it is evident that our upper limits indicate an evolution of the LF from $z=6$, even if only 170 Myr have passed since then. In the no-evolution case, in fact, we would expect to detect 5.5 objects, with a Poisson probability of no detection smaller than $0.5 \%$. This is confirmed by the results in Bouwens et al. (2006) who detected 7 objects at $z=6$ in the same luminosity range in the same field, in good agreement with our expectations.

Using a $C L=90 \%$ and assuming density evolution of the $\mathrm{LF}$, we found that the normalization of the LF at $z=7$ must be at most $40 \%$ of that at $z=6$. The evolution of the LF from 
$z=7$ to $z=6$ could also be in luminosity rather than density, corresponding to a brightening of the objects with cosmic time rather than to an increase in their number. This could be a better approximation of the real evolution if the systems at higher redshifts tend to be smaller or less active than the corresponding systems at lower redshift. In this case the minimum evolution of the average luminosity compatible with our upper limits at $\mathrm{CL}=$ $90 \%$ is about $0.22 \mathrm{mag}$. This evolution corresponds to a reduction of $20 \%$ in the total SFD, obtained by integrating the LF assuming a constant faint-end slope $\alpha$.

Very similar results are obtained if the $\mathrm{LF}$ at $z=6$ by Bunker et al. (2004) is used as a reference point. By analyzing a much smaller sample of LBGs, these authors found that the shift in the LF between $z=3$ and $z=6$ is consistent with density rather than luminosity evolution. Using this determination, the ratio $\operatorname{SFD}(z=7) / \operatorname{SFD}(z=6)$ is about 0.3 for the luminosity evolution and 0.8 for the density evolution.

Our limits can be compared with the LF at $z=3$ (Steidel et al. 1999). Assuming luminosity evolution we obtain a shift of $L^{*}$ about $0.9 \mathrm{mag}$, corresponding to $\operatorname{SFD}(z=7) / \operatorname{SFD}(z=$ $3)=0.42$, a reduction of more than a factor of 2 . A pure density evolution would require $\operatorname{SFD}(z=7) / \operatorname{SFD}(z=3)=0.05$.

A strong reduction in the $\mathrm{LF}$ from $z=6$ to $z=7$ is consistent with the tentative detection of one $z^{\prime}$-dropout LBG at $z=7$ in the HUDF by Bouwens et al. (2004b) as revised by Bouwens \& Illingworth (2006).

\section{The evolution of the star formation activity}

These findings on the relative evolution of the SFD can be compared with the results from other studies at lower and higher redshifts. The UV luminosity density $\rho_{1500}$ can be converted to SFD by using the Madau et al. (1998) ratio. Our upper limits to the density of galaxies at $z=7$ with $(J+K s)<25.5$ can be directly converted into an upper limit to the SFD contained in galaxies with $M_{1500}<-21.44$, which turns out to be about $<30 \%$ of that at $z=6$ and $<5 \%$ of that at $z=3$. As a consequence, our data show a strong reduction in SF activity in bright galaxies.

In Fig. 8 we show the values of the SFD obtained by integrating the observed LFs above a given luminosity threshold. The most interesting quantity, the total SFD at each redshift, would be obtained by using a very low luminosity threshold. Unfortunately, this would introduce large uncertainties due to the unobserved part of the LF at low luminosities. For example, Steidel et al. (1999) observe their LBGs at $z=3$ down to $\sim 0.1 L^{*}$, while for their value of the faint-end slope of the $\operatorname{LF}(\alpha=-1.6)$, about half of the total SFD takes place in galaxies below this limit. This implies that a correction of about a factor of two is needed to obtain the total SFD from the observed part of the LF. As for $\alpha<-2.0$, the integral of the LF no longer converges, and this correction becomes even larger for more negative values of $\alpha$ : it is 2.4 for $\alpha=-1.73$ (as in Bouwens et al. 2006) and even 6 for $\alpha=-1.9$, the most negative value of $\alpha$ compatible with the $i$-dropouts in Bouwens et al. (2006). To avoid this additional uncertainty, it is common to refer to the SFD derived from galaxies that where observed directly or by using a small extrapolation of the LF. For the value at $z=7$, we plot the average between the two extremes of pure luminosity and pure density evolution, while the upper value corresponds to pure luminosity evolution.

The two panels of Fig. 8 show the SFD as obtained by integrating the observed LFs with two different lower limits. In the upper panel we show the results when considering, at any redshift, the same range of absolute magnitudes

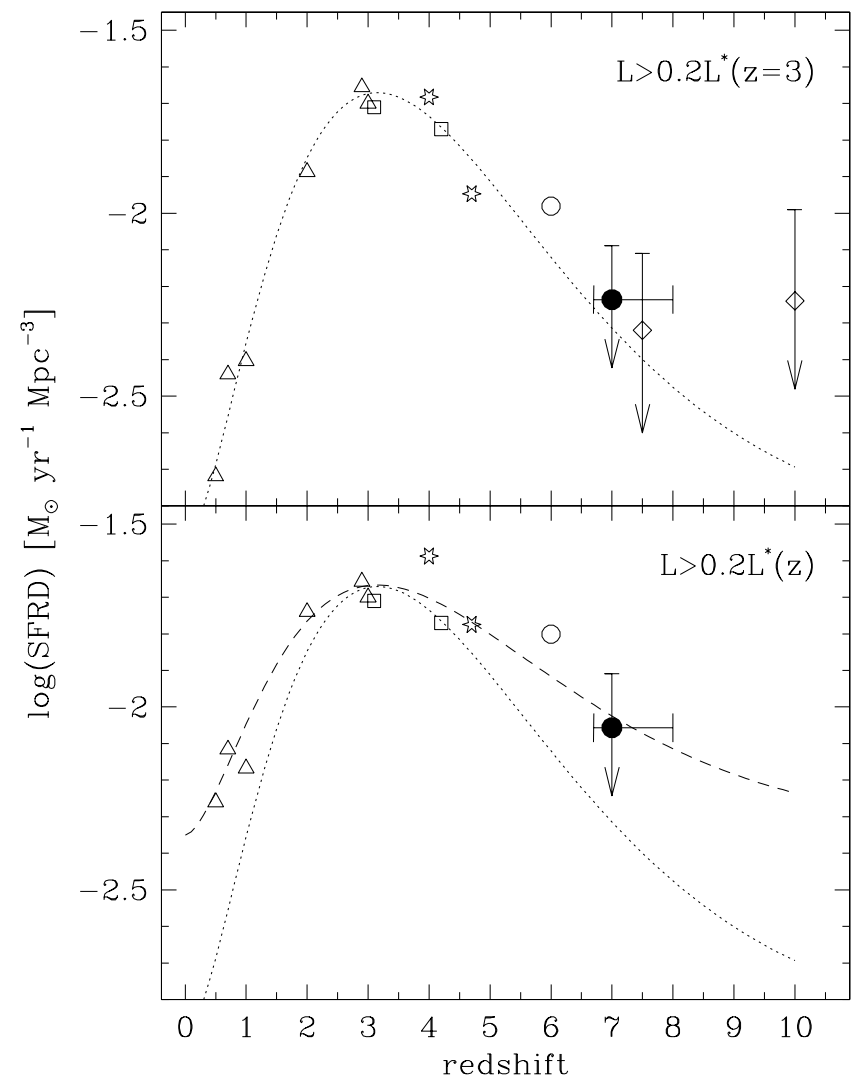

Fig. 8. Cosmic star formation as derived from rest-frame UV observations and with no correction for dust extinction. The upper panel shows the results of integrating the LF for $L>0.2 L^{*}(z=3)$, the lower panel for $L>0.2 L^{*}(z)$ (see text). The black dot with error bars is obtained from the upper limit from the present work, and the empty dots are obtained by integrating several published LFs (triangles: Arnouts et al. 2005; squares: Steidel et al. 1999; stars: Ouchi et al. 2004; circle: Bouwens et al. 2006) over the same interval. The diamonds at $z=7.5$ and $z=10$ are from Bouwens et al. (2004b, 2005) and are the only points not derived from an $L F$. The original published values referred to $L>0.3 L^{*}(z=3)$ and were corrected to $L>0.2 L^{*}(z=3)$ by assuming $\alpha=-1.73$ as observed at $z=6$. The dotted lines shows an empirical fit to the data in the upper panel, the dashed line to those in the lower panel.

$(M(1500)>-19.32)$, i.e., limiting the luminosity to above $20 \%$ of the $L^{*}$ magnitude derived by Steidel et al. (1999) for their LBGs at $z=3\left(L>0.2 L^{*}(z=3)\right)$. The derived SFD is directly related to the total SFD in the case of pure density evolution, as the fraction of SFD from galaxies below the threshold would be constant. The use of the absolute limits of integration is very common (see, for example, Bouwens et al. 2006; Shiminovich et al. 2005). The resulting SFD appears to vary rapidly, increasing by more than a factor of 30 from $z=0.3$ to $z=3$ and then decreasing by a factor of 2.2 to $z=6$ and $\sim 4$ to $z=7$.

In the case of luminosity evolution, the use of a constant limit of integration results in considering a variable fraction of LF. For example, Arnouts et al. (2005) measured the UV LF at low redshift and found a strong luminosity evolution, with $M^{*}=-18.05$ at $z=0.055$ and $M^{*}=-20.11$ at $z=1.0$. In this case, at low redshift the limit of integration used above $(M(1500)>-19.32)$ is even brighter than $L^{*}$ and only a small fraction of the LF is integrated to obtain a value of the SFD. This is the reason that the upper panel of Fig. 8 shows such a strong evolution at low redshift. In the lower panel of Fig. 8, we use a variable limit of integration, set to be $0.2 L^{*}(z)$ at each redshift. This is more 
Table 2. Values of the UV luminosity density and SFD at $z=7$, where the limits are at $90 \%$ confidence level.

\begin{tabular}{lcccc}
\hline \hline & \multicolumn{2}{c}{$L>0.2 L^{*}(z=3)$} & \multicolumn{2}{c}{$L>0.2 L^{*}(z)$} \\
& $\lg \rho^{a}$ & $\operatorname{lg~SFD}$ & $\lg \rho^{a}$ & $\operatorname{lg~SFD}$ \\
\hline$z=3^{c}$ & 26.17 & -1.72 & 26.17 & -1.72 \\
$z=6^{d}$ & 25.92 & -1.98 & 26.10 & -1.80 \\
$z=7$, lumin. evol. & $<25.76$ & $<-2.14$ & $<26.01$ & $<-1.89$ \\
$z=7$, dens. evol. & $<25.52$ & $<-2.37$ & $<25.70$ & $<-2.20$ \\
\hline
\end{tabular}

${ }^{a} \mathrm{erg} / \mathrm{s} / \mathrm{Hz} / \mathrm{Mpc}^{3}$ at $1500 \AA{ }^{b} M_{\odot} / \mathrm{yr} / \mathrm{Mpc}^{3}$; ${ }^{c}$ Steidel et al. (1999); ${ }^{d}$ Bouwens et al. (2006).

suitable to reproducing the total cosmic SFD, as the luminosity evolution appears to dominate both at low (Arnouts et al. 2005) and at high redshifts (Bouwens et al. 2006). In this case, the obtained evolution is much milder with an increase of a factor of 5 from $z=0.3$ to $z=3$ and a decrease of $\sim 1.4$ to $z=6$ and $\sim 2.5$ to $z=7$. The resulting values of the UV luminosity density and of the SFD are listed in Table 2 for both limits of integration.

\section{Discussion}

The search for galaxies at even higher redshift, $z>8$, by looking for $J$-dropout galaxies, faces another problem. The absence of deep enough data at wavelengths longer than $K$ s make it impossible to select galaxies with the standard color-color technique, and the selection relies on the $J-K$ s color alone. As a consequence, this technique is prone to the presence of many interlopers, and the constraints on the SFD at these redshifts are correspondingly weaker. Bouwens et al. (2005) looked for $z \sim$ 10 galaxies by selecting $J$-dropouts in a deep HST/NICMOS field down to $H \sim 28$. They detected 3 candidates, one of which is considered reliable. It is currently not possible to investigate the nature of these 3 objects in greater detail. This fact spoils the possibility of using this result to put constraints on the SFD at $z=10$, which is only constrained not to be larger than at $z=6$. For this reason our results are broadly consistent with these limits.

On the contrary, our results are not consistent with the high value of the SFD derived at $z=6-10$ by Richard et al. (2006). They measure a value of the SFD higher than at $z=3$, but they also warn against measuring SFD by using strongly clustered fields.

Even if the survey area is quite large for the obtained magnitude limits (25 times larger than the Hubble Deep Field, for example), the cosmic variance is still a potentially important concern. From the cross-correlation of the galaxies at this magnitude range it is possible to estimate (see, for example, Cresci et al. 2006) that the observed density can vary by about $20 \%$ of the cosmic average because of this effect. Similar results were obtained by Somerville et al. (2004) on the basis of cosmological simulations. As a consequence, cosmic variance is not expected to be a dominant effect.

All the data in Fig. 8 are derived from UV observations and, as a consequence, they are very sensitive to dust extinction, as discussed by a large number of authors (see, for example, Meurer et al. 1999). Variation in the dust content along the cosmic age is one of the effects that could contribute to shaping the observed evolution of the SFD. The typical color of $z=6$ LBGs, represented by the UV spectral slope $\beta$, is bluer at $z=6(\beta=-1.8$ according to Bouwens et al. (2006), revised to $\beta=-2.0$ by Bouwens \& Illingworth (2006) when using the new NICMOS zero points, and $\beta=-2.2$ for
Stanway et al. (2005)) than at $z=3(\beta=-1.1$, Meurer et al. 1997 , or $\beta=-1.5$, Adelberger \& Steidel 2000), pointing towards a reduction in average extinction at high redshift of about a factor of two. As a consequence, the observed reduction in the SFD cannot be due to an increase of the dust content. Actually, considering this effect would make the increase of SFD with cosmic time even more pronounced.

As we observe the bright part of the LF, we cannot exclude that the reduction in the number density of bright galaxies is not associated with an increase in that of the faint galaxies. If, for example, both $\Phi^{*}$ and $L^{*}$ vary together so that $\Phi^{*} L^{*}$ remains constant, the resulting total SFD also remains constant. We cannot exclude this effect, even if the upper limits to the galaxies with $L \sim L^{*}$ from Bouwens et al. (2004b) tend to exclude this possibility.

\section{Consequences on reionization of the primordial universe}

It is widely accepted that high redshift starburst galaxies can contribute substantially to the reionization of the universe. Madau et al. (1999) (see also Bunker et al. 2004) estimated the amount of star formation needed to provide enough ionizing photons to the intergalactic medium. By assuming an escape fraction of the photons $f_{\text {esc }}$ of 0.5 and a clumping factor $C$ of 30 (Madau et al. 1999), we find that at $z=7$ the necessary SFD is

$\mathrm{SFD}($ needed $) \sim 7.8 \times 10^{-2} M_{\odot} \mathrm{yr}^{-1} \mathrm{Mpc}^{-3}$.

The observed total amount of SFD derived from UV observations can be derived by integrating the observed LF down to zero luminosity. Assuming luminosity evolution and integrating the $\mathrm{LF}$ down to $0.01 L^{*}$, we obtain

$\mathrm{SFD}($ observed $)=2.9 \times 10^{-2} M_{\odot} \mathrm{yr}^{-1} \mathrm{Mpc}^{-3}$

where about half of this comes in very faint systems, below $0.1 L^{*}$. This value can be be increased up to $\sim 5 \times$ $10^{-2} M_{\odot} / \mathrm{yr}^{-1} \mathrm{Mpc}^{-3}$ assuming the steeper faint-end slope on the LF ( $\alpha=-1.9)$ compatible with the data in Bouwens et al. (2006).

The uncertainties involved in this computation (as the actual values of $f_{\text {esc }}$ and $C$, the amount of evolution of the LF between $z=6$ and $z=7$, the faint-end slope $\alpha$ of the LF) are numerous and large for both SFD(needed) and SFD(observed). Also, Stiavelli et al. (2004) discuss how very metal-poor stars can overproduce ionizing photons and how a warmer IGM could lower the required flux. Nevertheless, the amount of ionizing photons that can be inferred at $z=7$ from observations is less or, at most, similar to the needed value. A measure of the SFD at even higher redshifts or tighter constraints to the faint-end slope of the LF at $z=6$ can significantly reduce these uncertainties and can easily reveal that it falls short of the required value.

\section{Conclusions}

The existing multi-wavelength deep data on the large GOODSSouth field allowed us to search for $z=7$ star-forming galaxies by selecting $z^{\prime}$-dropouts. The accurate study of the dropouts in terms of colors, morphology, and spectra allows us to exclude the presence of any $z=7$ galaxy in the field above our detection threshold. We used this to derive evidence for the evolution of the LF from $z=7$ to $z=6$ to $z=3$, and to determine an upper limit to the global star formation density at $z=7$. These limits, together with the numerous works at lower redshifts, point 
toward the existence of a sharp increase of the star formation density with cosmic time from $z=7$ to $z=4$, a flattening between $z=4$ and $z=1$, and a decrease afterward. The ionizing flux from starburst galaxies at $z=7$ could be too low to produce all the reionization.

The first star forming systems at $z=7$ appear to be too faint to be studied in detail with the current generation of telescopes, and the detailed comprehension of their properties will be possible when the telescopes of the next generation come into use.

Acknowledgements. We are grateful to R. Bouwens for useful discussion and for having provided the catalog of his $i$-dropouts before publication, and to the referee, A. Bunker, for very useful comments on the first version of the paper. We also thank the Paranal staff for service observing and assistance during visitor observations, and L. Testi for useful discussions about brown dwarfs.

\section{References}

Adelberger, K. L., \& Steidel, C. C. 2000, ApJ, 544, 218

Allard, F., Hauschildt, P. H., Alexander, D. R., Tamanai, A., \& Schweitzer, A. 2001, ApJ, 556, 357

Alvarez, M. A., Shapiro, P. R., Ahn, K., \& Iliev, I. T. 2006, ApJ, 644, L101

Arnouts, S., Shiminovich, D., Ilbert, O., et al. 2005, ApJ, 619, L43

Becker, R. H., Fan, X., White, R. L., et al. 2001, AJ, 122, 2850

Bertin, E., \& Arnouts, S. 1996, A\&AS, 117, 393

Bolzonella, M., Miralles, J.-M., \& Pell, R. 2000, A\&A, 363, 476

Bouwens, R. J., Illingworth, G. D., Thompson, R. I., et al. 2004a, ApJ, 606, 25

Bouwens, R. J., Thompson, R. I., Illingworth, G. D, et al. 2004b, ApJ 616, 79

Bouwens, R. J., Illingworth, G. D., Thompson, R. I., \& Franx, M. 2005, ApJ, 642,5

Bouwens, R. J., \& Illingworth, G. D. 2006, Nature, 443, 189

Bouwens, R. J., Illingworth, G. D., Blakeslee, J. P., \& Franx, M. 2006, ApJ, in press [arXiv:astro-ph/0509641]

Bruzual, G., \& Charlot, S. 2003, MNRAS, 344, 1000

Bunker, A., Stanway, E., Ellis, R., McMahon, G., \& McCarthy, P. J. 2003, MNRAS, 342, L47

Bunker, A., Stanway, E., Ellis, R., \& McMahon, G. 2004, MNRAS, 355, 374

Cardelli, J. A., Clayton, G. C., \& Mathis, J. S. 1989, ApJ, 345, 245

Cen, R., Haiman, Z., \& Mesinger, A. 2005, ApJ, 621, 89

Cowie, L. L., Songaila, A., Hu, E. M., \& Cohen, J. G. 1996, AJ, 112, 839

Cresci, G., et al. 2006, in press

Dawson, S., Stern, D., Bunker, A. J., Spinrad, H., \& Dey, A. 2001, AJ, 122, 598

Dickinson, M., Stern, D., Giavalisco, M., et al. 2004, ApJ, 600, L99

Dunlop, J. S., Cirasuolo, M., \& McLure, R. J. 2006, MNRAS, submitted [arXiv: astro-ph/0606192]

Eyles, L., Bunker, A., Ellis, R., et al. 2005, MNRAS, 364, 443

Eyles, L., Bunker, A., Ellis, R., et al. 2006, submitted

[arXiv:astro-ph/0607306]
Fan, X., Narayanan, V. K., Lupton, R. H., et al. 2001, AJ, 122, 2833 Fan, X., Narayanan, V. K., Strauss, M. A., et al. 2002, AJ, 123, 1247 Fan, X., Strauss, M. A., Schneider, D. P., et al. 2003, AJ, 125, 1649 Francis, P. J., \& Koratkar, A. 1995, MNRAS, 274, 504 Giavalisco, M. 2002, ARA\&A, 40, 579

Giavalisco, M., Ferguson, H. C., Koekemoer, A. M., et al. 2004a, ApJ, 600, L93 Giavalisco, M., Dickinson, M., Ferguson, H. C., et al. 2004b, ApJ, 600, L103 Gnedin, N., \& Prada, F. 2004, ApJ, 608, L77

Grazian, A., Fontana, A., De Sanctis, C., et al. 2006, A\&A, 453, 507

Gunn, J. E., \& Peterson, B. A. 1965, ApJ, 142, 1633

Hopkins, A. M. 2004, ApJ, 615, 209

Hopkins, A. M., \& Beacom, J. F. 2006, ApJ, 651, 142

Hu, E. M., \& McMahon, R. G. 1996, Nature, 382, 231

Hu, E. M., McMahon, R. G., Cowie, L. L., et al. 1999, ApJ, 522, L9

Hu, E. M., Cowie, L. L., McMahon, R. G., et al. 2002, ApJ, 568, L75

Kodaira, K., Taniguchi, Y., Kashikawa, N., et al. 2003, PASP, 55, L17

Leggett, S. K., Golimowski, D. A., Fan, X., et al. 2002, ApJ, 564, 452

Labbé, I., Franx, M., Rudnick, G., et al. 2003, AJ, 125, 1107

Le Fèvre, O., Paltani, S., Arnouts, S., et al. 2005, Nature, 437, 519

Loeb, A., Barkana, R., Hernquist, L. 2005, ApJ, 620, 553

Madau, P., Pozzetti, L., Dickinson, M., et al. 1998, ApJ, 498, 106

Madau, P., Haardt, F., \& Rees, M. 1999, ApJ, 266, 713

Maiolino, R., Oliva, E., Ghinassi, F., et al. 2004, A\&A, 420, 889

Malhotra, S., \& Rhoads, J. E. 2002, ApJ, 565, L71

Mannucci, F., Basile, F., Poggianti, B. M., et al. 2001, MNRAS, 326, 745

Meurer, G. R., Heckman, T. M., Lehnert, M. D., Leitherer, C., \& Lowenthal, L. 1997, AJ, 114, 54

Meurer, G. R., Heckman, T. M., \& Calzetti, D. 1999, ApJ, 521, 64

Mobasher, B., Dickinson, M., Ferguson, H. C., et al. 2005, ApJ, 635, 832

Ouchi, M., Shimasaku, K., Okamura, S., et al. 2004, ApJ, 611, 660

Paltani, S., Le Fevre, O., Ilbert, O., et al. 2006, A\&A, in press [arXiv:astro-ph/0608176]

Pentericci, L., Fan, X., Rix, H.-W., et al. 2002, AJ, 123, 2151

Richard, J., Pelló, R., Schaerer, D., Le Borgne, J.-F., \& Kneib, J.-P. 2006, 456, 861

Ricotti, M., Haehnelt, M. G., Pettini, M., \& Rees, M. J. 2004, MNRAS, 352, L21

Shiminovich, D., Ilbert, O., Arnouts, S., et al. 2005, ApJ, 619, L47

Shimasaku, K., Ouchi, M., Furusawa, H., et al. 2005, PASJ, 57, 447

Somerville, R. S., Lee, K., Ferguson, H. C., et al. 2004, ApJ, 600, L171

Spinrad, H., Stern, D., Bunker, A., et al. 1998, AJ, 116, 2617

Stanway, E. R., Bunker, A. J., \& McMahon, R. G. 2003, MNRAS, 342, 439

Stanway, E. R., Glazebrook, K., Bunker, A. J., et al. 2004a, ApJ, 604, L13

Stanway, E. R., Bunker, A. J., McMahon, R. G., et al. 2004b, ApJ, 607, 704

Stanway, E. R., McMahon, R. G., \& Bunker, A. J. 2005, MNRAS, 359, 1184

Steidel, C. C., Giavalisco, M., Pettini, M., et al. 1996, ApJ, 462, L17

Steidel, C. C., Adelberger, K. L., Giavalisco, M., et al. 1999, ApJ, 519, 1

Steidel, C. C., Adelberger, K. L., Shapley, A. E., et al. 2003, ApJ, 592, 728

Stiavelli, M., Fall, M., \& Panagia, N. 2004, ApJ, 610, 1

Taniguchi, Y., Ajiki, M., Nagao, T., et al. 2005, PASP, 57, 165

Testi, L., D’Antona, F., Ghinassi, F., et al. 2001, ApJ, 552, 147 